\title{
Molecular profiling of thyroid cancer subtypes using large-scale text mining
}

\author{
Chengkun Wu $\mathbf{W}^{1,2,3}$, Jean-Marc Schwartz', Georg Brabant ${ }^{4,5}$, Goran Nenadic ${ }^{3,6,7^{*}}$ \\ From Asia Pacific Bioinformatics Network (APBioNet) Thirteenth International Conference on Bioinformatics \\ (InCoB2014) \\ Sydney, Australia. 31 July - 2 August 2014
}

\begin{abstract}
Background: Thyroid cancer is the most common endocrine tumor with a steady increase in incidence. It is classified into multiple histopathological subtypes with potentially distinct molecular mechanisms. Identifying the most relevant genes and biological pathways reported in the thyroid cancer literature is vital for understanding of the disease and developing targeted therapeutics.

Results: We developed a large-scale text mining system to generate a molecular profiling of thyroid cancer subtypes. The system first uses a subtype classification method for the thyroid cancer literature, which employs a scoring scheme to assign different subtypes to articles. We evaluated the classification method on a gold standard derived from the PubMed Supplementary Concept annotations, achieving a micro-average F1-score of 85.9\% for primary subtypes. We then used the subtype classification results to extract genes and pathways associated with different thyroid cancer subtypes and successfully unveiled important genes and pathways, including some instances that are missing from current manually annotated databases or most recent review articles.

Conclusions: Identification of key genes and pathways plays a central role in understanding the molecular biology of thyroid cancer. An integration of subtype context can allow prioritized screening for diagnostic biomarkers and novel molecular targeted therapeutics. Source code used for this study is made freely available online at https:// github.com/chengkun-wu/GenesThyCan.
\end{abstract}

\section{Background}

Thyroid cancer (TC) is the most common endocrine malignancy [1] and its incidence increase has been significant in recent years despite some controversies about the extent [2]. Many possible factors causing thyroid cancer have been reported including exposure to ionising radiation, iodine-deficiency and heredity [3]. Conventional treatment strategies include surgical resection, radiation therapy (especially radioactive iodine therapy), chemotherapy and thyroid hormone therapy [4]. However, the understanding of the underlying molecular mechanisms is still incomplete.

\footnotetext{
* Correspondence: goran.nenadic@manchester.ac.uk

${ }^{3}$ Manchester Institute of Biotechnology, 131 Princess Street, Manchester M1 7DN, UK

Full list of author information is available at the end of the article
}

Thyroid tumours are usually classified into multiple subtypes according to their histopathological characteristics, and treatments are selected depending on the subtype and stage of thyroid cancer. The main subtypes include papillary thyroid cancer (PTC), follicular thyroid cancer (FTC), anaplastic thyroid cancer (ATC) and medullary thyroid cancer (MTC) [4]. PTC and FTC are also sometimes collectively referred to as differentiated thyroid cancer (DTC) or well-differentiated thyroid cancer (WDTC), while ATC can also be referred to as undifferentiated thyroid cancer. In addition, a number of rare subtypes have been described. Cellular origins and some of the known molecular mechanisms differ for each subtype [5], which may include subtype-specific alterations in DNA methylation patterns [3] and have led to new therapeutic approaches based on the molecular signature of the tumours. 
These "targeted therapeutics" of thyroid cancer are being rapidly developed [6,7]. Several potential drugs are currently in preclinical testing or in clinical use $[8,9]$. However, lack of systematic studies of underlying molecular mechanisms can lead to a high risk for thyroid cancer patients, who might suffer from unexpected side effects. For instance, RET has been shown to be an oncogene in thyroid cancer but is considered as a potential tumour suppressor gene in colorectal cancer [10]. Consequently, studies focusing on one or a few genes are likely to miss the molecular context that could be vital for a comprehensive understanding of the disease.

For systematic studies, a major challenge is to efficiently utilise the myriad of knowledge and information from unstructured scientific literature. PubMed, one of the most widely used systems for biomedical literature search [11], returns over 50,000 results with the search query 'thyroid cancer'. The number is increasing rapidly, with over 2,000 articles published annually in recent years, as illustrated in Figure 1. This trend has made it extremely difficult for scientists to identify, retrieve and assimilate all relevant publications.

To support the identification and retrieval of relevant articles, MeSH (Medical Subject Headings, http://www. nlm.nih.gov/pubs/factsheets/mesh.html) terms are used in PubMed to label the topic of each document, along with additional headings known as Supplementary Concepts (SCs). The MeSH term used for thyroid cancer is 'thyroid neoplasms', and the major subtypes are represented as SCs. However, the thyroid cancer SCs were only introduced in August 2010, which means the majority of the thyroid cancer literature does not have a subtype label indicated by SC.

Text mining techniques have been extensively used to support access to the biomedical literature [12,13]. A number of tasks can now be successfully fulfilled by text mining, including gene mention recognition and normalization $[14,15]$, pathway mention recognition [16] and event extraction $[17,18]$. Those efforts enable the extraction of structured and explicit data, and this further targets the exploration and investigation of specific biological questions.

In this paper we focus on identifying genes and pathways that are reported in the thyroid cancer literature, grouped by subtypes. We then compare our systematic effort to some established databases and demonstrate how our results could boost the understanding of thyroid cancer and promote comprehensive investigation for potential therapeutic targets.

\section{Related work}

There are several curated databases that contain molecular information of thyroid cancer. For example, the Thyroid Cancer and Disorder Gene Database (TCGDB, http://www.juit.ac.in/attachments/tcgdb/) contains information about genes and miRNAs involved in different thyroid cancer subtypes. The data in TCGDB have been extracted through manual literature review of a few selected articles. However, details of these articles are not revealed, so it is not clear how systematic and comprehensive the database is.

MalaCards is a generic integrated compendium for diseases and associated annotations [19], with some information related to TC. It provides disease-specific information from multiple sources, including related publications, genes and pathways. However, there are several limitations: firstly, the publications associated with a disease are obtained by title search only, which could miss out many relevant articles; secondly, pathways are provided by affiliated genes rather than their mentions in the literature.

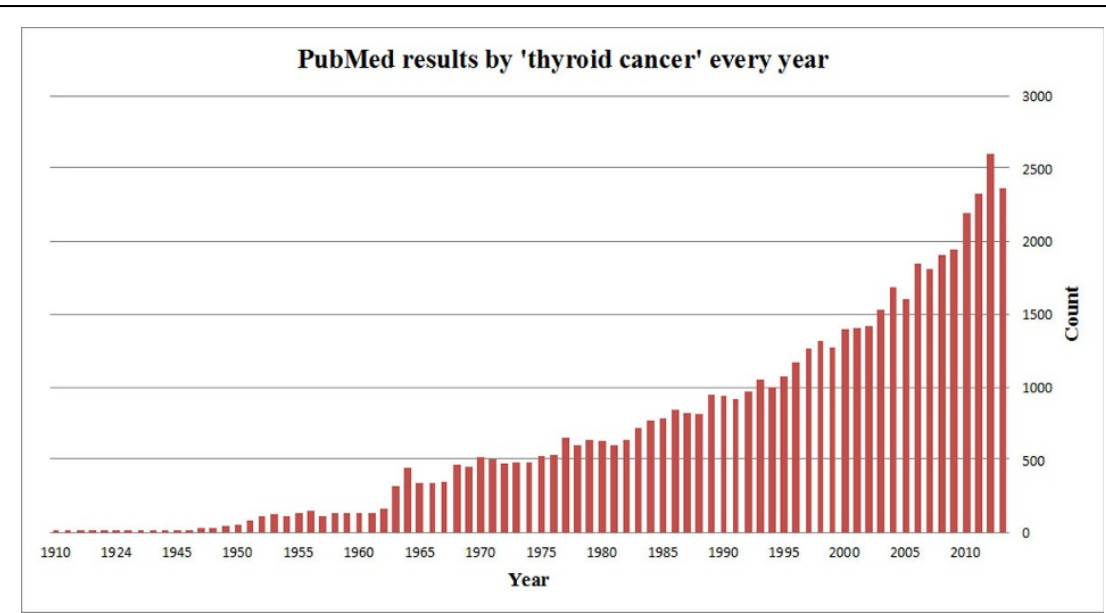

Figure 1 Number of PubMed articles returned by the 'thyroid cancer' query. The size of thyroid cancer related literature is increasing rapidly, with over 2,000 articles published annually in recent years. 
Finally, the coverage of this data set is limited: for example, a search for "anaplastic thyroid cancer" returns no affiliated genes.

Gene2Pubmed [20] is a generic database maintained by NCBI that provides a set of manually added links from PubMed articles to genes. Gene2Pubmed integrates information from a number of public databases and the links are not limited to articles specifically defining the function of a given gene. However, similarly to other resources, its coverage is limited: for example, we found quite a few examples where TC-related genes were missing from the Gene2Pubmed annotation (see Results).

Several databases contain information about pathways and their associations to diseases. KEGG [21], for example, contains pathways for different diseases, but its coverage is often limited, in particular for diseases that are not widely studied (e.g. there is a single pathway diagram for thyroid cancer). Even for databases that focus on specific diseases (e.g. AlzPathway [22] is a database of pathways associated to Alzheimer's disease) it is often difficult to keep up with new findings through manual curation, and automated text mining approaches need to be used to ensure a wider coverage [16].

There are various efforts to automatically extract relationships between cancer and its molecular bases from the literature. For instance, a maximum entropy-based named entity recognizer and relation recognizer were applied to find relations between prostate cancer and genes [23]. Other relation finding tools can be applied to cancer related studies as well, including FACTA [24], CoPub [25], DigSee [26] and OncoSearch (http://oncosearch.biopathway.org). Both FACTA and CoPub support searching for co-occurring biomedical concepts (genes/diseases), facilitated by indexing through dictionary matching or regular expression matching. DigSee and OncoSearch can scan the literature to identify whether a gene is up-regulated or down-regulated in a particular type of cancer. The systems look for genes that are reported together with a biological event and cancer in the same sentence. While the results are therefore often highly accurate, they may not be comprehensive. However, no systems could support extraction of information specific to thyroid cancer subtypes.

\section{Methods}

\section{Pipeline for generating the molecular profiling}

The pipeline we engineered to generate the molecular profiling of thyroid cancer subtypes is depicted in Figure 2. It starts with the corpus construction and is followed by the text mining tasks implemented as annotators within TextPipe [18], a framework designed for large-scale, modular text processing and data integration. Text mining results are then used to find the association between genes/pathways and thyroid cancer subtypes. These stages are explained in the following subsections.

\section{Data - a corpus of thyroid cancer from MEDLINE}

All the documents in our corpus were retrieved from MEDLINE. We performed a PubMed search with the query "((thyroid neoplasms[majr] AND human[mh] AND english[la]) OR thyroid[ti]) AND (cancer OR carcinoma OR malignant OR malignancy))", as suggested by the National Cancer Institute for retrieving the TC-relevant literature (see http://www.cancer.gov/cancertopics/litsearch/endocrine). It should be noted that the query limits the results to human studies in English. A total of 38,572 PubMed IDs (PMIDs) were fetched from PubMed (accessed on 13/01/2014). The complete list of PMIDs is available in Additional File 1. We retrieved abstracts from PubMed using those PMIDs and constructed the thyroid cancer corpus (TC_TEXT) using PyPubmedText, which is freely available from https://github.com/chengkun-wu/ PyPubmedText.

For the evaluation, we created the SC_GOLD corpus, which consists of all 1,213 documents with specified subtype SCs since their introduction in 2010 (see Additional File 2).

\section{Subtype classification using subtype scoring}

For subtype classification, we aimed to label the articles in the TC_TEXT corpus with the major TC subtype(s) discussed in the associated abstract. The subtypes we investigated included PTC, ATC, FTC and MTC. We considered this as a document classification task and have developed an efficient classification method based on a subtype-scoring scheme. Although machine-learning methods are popular for document classification [27], we did not employ them here due to insufficient training data for each of the TC subtypes: while the recent articles with SCs can be used as the training data, the number of documents is still limited (1,213 in total, much fewer for each subtype). Instead, we used a flexible dictionary matching method as described below.

For each article in the corpus, we calculate a vector of scores corresponding to each TC subtype through the following steps:

1. Subtype names are often mentioned partially and in abbreviated forms, due to language economy and flexibility (e.g. "...Inflammatory infiltrates could increase the risk of papillary cancer in patients with autoimmune lymphocytic thyroiditis..." (PMID: 21042739)). We therefore dissociate each subtype name into three parts: (1) subtype keyword (SK); (2) anatomy keyword (AK); (3) malignancy keyword (MK). For instance, in "papillary thyroid cancer", the 


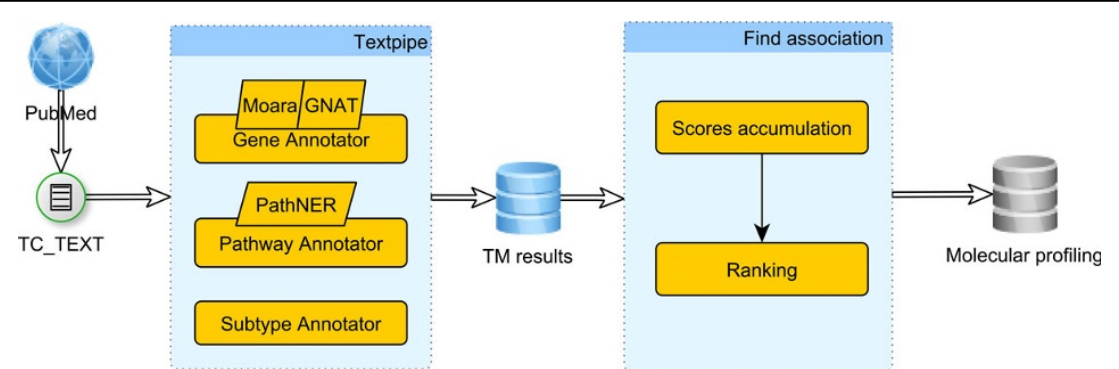

Figure 2 The pipeline to generate the molecular profiling of thyroid cancer subtypes. The pipeline starts with corpus construction, which is followed by text mining annotators implemented within the TextPipe framework. The text mining results are then used to find genes/ pathways association with thyroid cancer subtypes, which lead to a comprehensive molecular profiling of primary thyroid cancer subtypes.

SK is "papillary", the AK is "thyroid" and the MK is "cancer". We then match those parts against text separately, maintaining the three separate keyword lists for matching: a subtype keyword list, an anatomy keyword list and a malignancy keyword list. For each list, possible variants are also included. For instance, "anaplastic thyroid cancer" is also named as "undifferentiated thyroid cancer", which can be written as "un-differentiated thyroid cancer". So "anaplastic", "un-differentiated" and "undifferentiated" will all be mapped to "anaplastic". The lists are included in the additional files. For matching, we applied the open-source package LINNAEUS [28].

2. Each input document is split into sentences. For each sentence, we apply dictionary matching to calculate the subtype relevance scores of that sentence calculated as follows: (1) if SK co-occurs within that sentence with both AK and MK, then the sentence has a relevance score 1 to the subtype SK; (2) if SK co-occurs with only AK or MK, then the sentence has a relevance score 0.5 to the subtype SK; (3) if SK appears alone without the presence of either AK or $\mathrm{MK}$, the sentence has a relevance score 0.25 to the subtype SK. We defined these rules to cover the situations where subtype names are simplified, as illustrated above.

3. The vector of subtype scores $S_{i}=\left\langle S_{i}^{P}, S_{i}^{A}, S_{i}^{F}, S_{i}^{M}\right\rangle$ for article $d_{i}$ is calculated by weighted accumulation of the vectors of subtype scores for each sentence (the four elements in $S_{i}$ correspond to PTC, ATC, FTC and MTC respectively). The weights of different sentences are assigned in the following way: the title of a document is considered as the most important element and is assigned a weight of 4; the first sentence in the abstract usually mentions the main topic of the document and the last sentence usually concludes the article, and both are assigned a weight of 2; the second and the penultimate sentence can be quite important as well, and are both assigned a weight of 1 ; other sentences in the abstract are given a weight of 0.5 in order to weaken bypassing mentions of subtype names that are not the major scope of the article.

For classification, we set threshold values for each subtype and assign the corresponding label to the article if the associated subtype score is above a pre-set value. We assigned slightly different thresholds to different subtypes, with the PTC's percentage threshold slightly higher than other subtypes, given that PTC occurs more frequently in the literature (over 50\%).

\section{Gene recognition and normalisation}

A number of tools are available for identifying mentions of genes in the literature and normalising them to database identifiers. We utilized two open source libraries, Moara [14] and GNAT [15], which have been successfully applied in other studies $[18,26]$. For gene name recognition, Moara utilizes the CBR-tagger [29], which treats the recognition problem as a binary classification on each token; GNAT employs dictionary-expanded regular expressions together with BANNER [30]. For the normalisation of recognized gene names, both tools map mentions to the Entrez Gene database [20], adopting similar methods for disambiguation. We wrapped Moara and GNAT as TextPipe annotators that output normalised gene mentions including document ID (PubMed ID), positions in the text, normalised Entrez Gene ID, original text and the applied tool (Moara or GNAT). If the two tools report overlapping mentions, we created a new one covering both mentions (union); if the overlapping mentions have been assigned different Entrez Gene identifiers, the priority is given to GNAT as its reported performance is higher.

\section{Pathway mention recognition}

We utilised PathNER for pathway mention recognition from the literature [16]. PathNER is implemented using soft dictionary matching and manually created rules. For this study, we recompiled the pathway dictionary using 
the data from the 2013 update of the ConsensusPathDB interaction database [31]. The outputs from this annotator are biological pathway mentions including document ID (PubMed ID), positions in the text, pathway database identifier (if available) and original text.

\section{Association between genes/pathways and subtypes}

To establish the association between text-mined information and thyroid cancer subtypes, we performed the following steps for each text-mined entity (gene or pathway, referred to as E thereafter):

1. Get all the documents $D_{E}=\left\{d_{i} \mid i \in[1, n]\right\}$ that mention $\mathrm{E}$ according to the text-mining results; 2. For each document $d_{i} \in D_{E}$, get the subtype score vector $S_{i}=\left\langle S_{i}^{P}, S_{i}^{A}, S_{i}^{F}, S_{i}^{M}\right\rangle$ as described above;

3. Sum up all $S_{i}$ to give the subtype relevancy vector of $\mathrm{E}$ :

$$
S_{E}=\sum_{i=1}^{n} S_{i}=\left\langle\sum_{i=1}^{n} S_{i}^{P}, \sum_{i=1}^{n} S_{i}^{A}, \sum_{i=1}^{n} S_{i}^{F}, \sum_{i=1}^{n} S_{i}^{M}\right\rangle=\left\langle S_{E}^{P}, S_{E}^{A}, S_{E}^{F}, S_{E}^{M}\right\rangle
$$

For a given subtype $j \in\{P, A, F, M\}$, we generate a list of entities $L_{j}$, which is composed of entities that have at least one document labelled as subtype $j$ in the classification stage; we then rank the entities in $L_{j}=\left[E_{1}, E_{2}, \ldots, E_{k}\right]$ in a descending order by the value of $S_{E}^{j}$ for each entity $E$ in $L_{j}$.

\section{Results and discussion}

\section{Subtype classification evaluation}

For performance assessment against the gold standard, we adopted the standard metrics: Precision (P), Recall $(R)$ and F1-score (F1) defined by the following equations:

$$
P=\frac{T P}{T P+F P}, \quad R=\frac{T P}{T P+F N}, \quad F 1=2 \frac{P \cdot R}{P+R}
$$

Here, $T P$ is the number of true positives, FP is the number of false positives and $F N$ is the number of false negatives. Those metrics were calculated for each subtype. For documents with multiple subtype labels, the labels were evaluated separately.

The evaluation of the classification performance against the SC_GOLD corpus for each TC subtype is listed in Table 1. The performance for PTC, ATC and MTC shows good precision and moderate recall, with F1-score over $85 \%$. This demonstrates the effectiveness of our scoring scheme.

Unexpectedly, the precision for FTC was low (17.5\%). We therefore performed error analysis to investigate the reason for that. We found that more than half of all errors (54 of the 99 FPs for FTC) are from articles about DTC or WDTC, referring to both PTC and FTC [32]. However, it seems that the PubMed SCs typically only annotate an article with PTC if it is about DTC.
Table 1 TC subtype classification performance on the SC_GOLD corpus.

\begin{tabular}{lllllllll}
\hline & & TP & TN & FN & FP & Precision & Recall & F1 \\
\hline $\begin{array}{c}\text { Our } \\
\text { Method }\end{array}$ & PTC & 641 & 372 & 186 & 14 & 0.979 & 0.775 & 0.865 \\
& & & & & & & & \\
& ATC & 114 & 1066 & 27 & 6 & 0.950 & 0.809 & 0.874 \\
& FTC & 21 & 1081 & 12 & 99 & 0.175 & 0.636 & 0.275 \\
& FTC* & 75 & 1081 & 12 & 45 & 0.625 & 0.862 & 0.725 \\
& MTC & 189 & 979 & 41 & 4 & 0.979 & 0.822 & 0.894 \\
& Micro & 1019 & 3498 & 266 & 69 & 0.937 & 0.793 & 0.859 \\
& Average & & & & & & & \\
\hline Baseline & & TP & TN & FN & FP & Precision & Recall & F1 \\
& PTC & 601 & 372 & 226 & 14 & 0.977 & 0.727 & 0.834 \\
& ATC & 109 & 1063 & 32 & 9 & 0.924 & 0.773 & 0.842 \\
& FTC & 19 & 1061 & 14 & 119 & 0.320 & 0.485 & 0.386 \\
& FTC* & 73 & 1061 & 14 & 65 & 0.529 & 0.839 & 0.649 \\
& MTC & 181 & 971 & 49 & 12 & 0.938 & 0.787 & 0.856 \\
& Micro & 964 & 3467 & 321 & 100 & 0.906 & 0.750 & 0.821 \\
& Average & & & & & & & \\
\hline
\end{tabular}

FTC* is the result after adjusting the results by considering WDTC. The microaverage calculation uses FTC*.

This is probably because the SC for FTC was introduced in August 2012, while the SC for PTC was introduced two years earlier. If those 54 cases are considered TPs then the performance is obviously improved (F1 is $72.5 \%$; listed in Table 1 as FTC*). We also found a few other variants such as "follicular variant papillary carcinoma", "mixed medullary-follicular carcinoma of the thyroid", "follicular variant of PTC", etc. We checked the frequency of those variants by dictionary matching and we found them in 31 documents in SC_GOLD. As our classification "dissects" thyroid cancer subtype names into several parts, follicular variants will score and get incorrectly classified as FTC in those situations. The FTC precision is thus more affected as "follicular" is a word commonly used in PTC articles, since PTCs are derived from follicular cells [33].

In Table 1, we also listed the performance of a baseline method for comparison. The baseline method performs classification based on subtype name occurrences in titles and abstracts by dictionary matching using LINNAEUS [28]. The dictionary includes all available synonyms for the TC subtype names. The performance results demonstrate that our method outperforms the baseline for all subtypes.

\section{Application to TC_TEXT}

We ran our classification method on the whole TC_TEXT corpus (38,572 abstracts). The complete classification results (with subtype relevancy scores and predicted labels) can be found in Additional File 3. The number of articles labelled with each subtype is listed in Table 2, which also shows the incidence rate of each 
Table 2 Number of articles for each subtype in the TC_TEXT corpus.

\begin{tabular}{ccccccc}
\hline & PTC & ATC & FTC & MTC & XTC* & TOTAL \\
\hline \#Articles & 7795 & 1122 & 4696 & 3316 & 3768 & 26866 \\
$\%$ & $29.0 \%$ & $4.2 \%$ & $17.5 \%$ & $12.3 \%$ & $14.0 \%$ & \\
Incidence $^{\S}$ & $75 \%$ & $1-2 \%$ & $10-15 \%$ & $5-10 \%$ & & \\
\hline
\end{tabular}

XTC*: represents articles that contain more than one subtype labels. Incidence ${ }^{\S}$ data source: PTC, FTC, ATC [2], MTC [39]

subtype. We can see that the amount of literature somewhat reflects the incidence rate, with PTC as the most frequent and ATC as the least frequent among the four subtypes studied here. The results also show that it is relatively uncommon to discuss more than one subtype in an article (see Figure 3), with the exception of PTC and FTC, which are frequently studied together as they are both derived from follicular cells [33].

\section{Gene normalization results and gene-subtype association}

We ran gene recognition and normalization on the TC_TEXT corpus and a total number of 2,833 unique genes were detected. We first compared the resulting list against the gene list generated from the Gene2Pubmed database [20]. Out of 914 genes identified in Gene2Pubmed, $762(83.4 \%)$ are found by our text mining approach. On the other hand, 2,071 out of 2,833 textmined genes $(73.1 \%)$ are not listed in the Gene2Pubmed database (see Figure 4). We randomly sampled 50 genes from that difference set and manually checked whether they are associated with TC or not. Table 3 shows these genes sorted by their document-level frequency, according to text mining results. If a gene is related to $\mathrm{TC}$, an example of relevant document is given. Overall, 42 genes out of

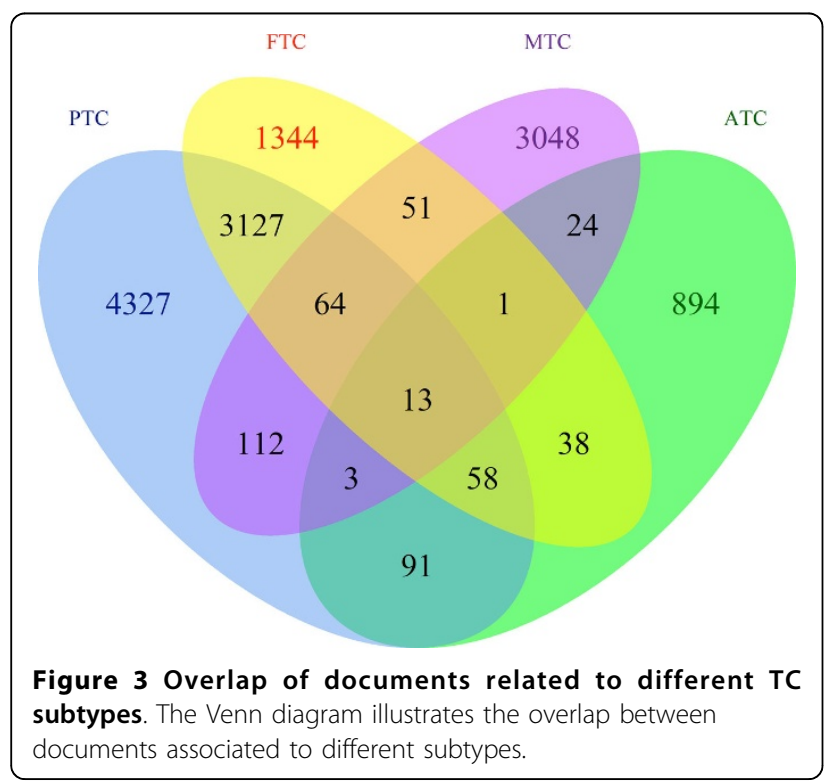

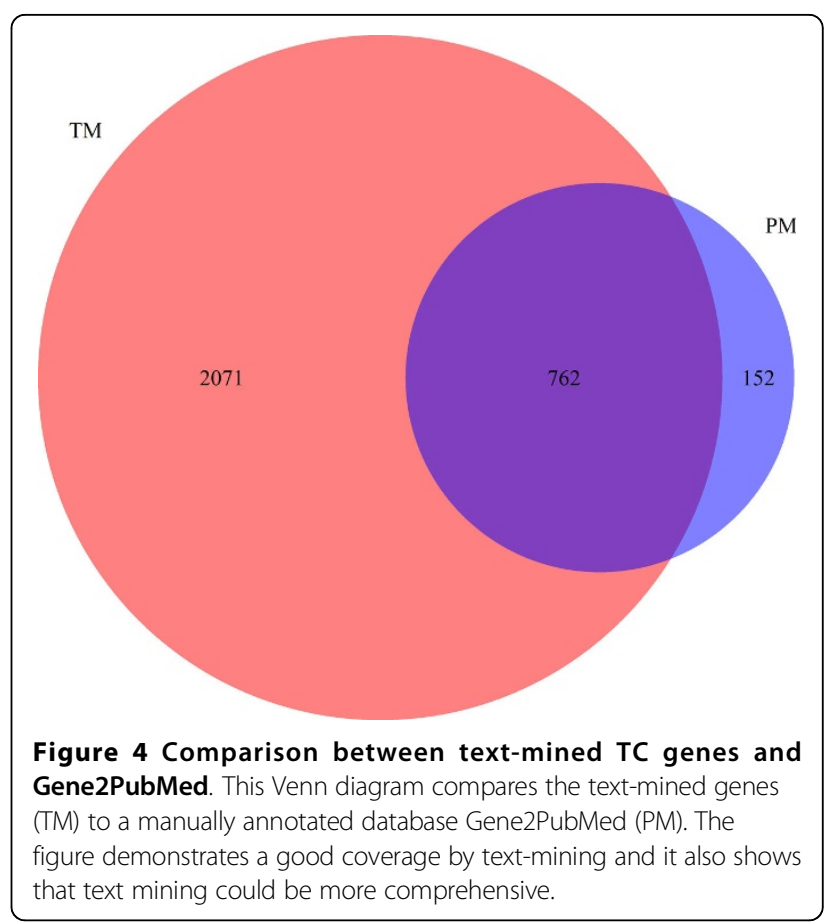

50 are found to be reported as associated with thyroid cancer. This highlights the importance of using text mining for a comprehensive retrieval of information, complementing manually annotated data, which may potentially miss over 1,700 TC-related genes ( $84 \%$ of 2,071$)$. We also note that even genes with low document-level frequencies can be relevant: for instance, WNT7A (Entrez Gene ID: 7476) was only detected once by text mining, but is reported to be over expressed in both PTC and its aggressive variant. Therefore, we did not use any filters based on documentlevel frequency in this study.

With the subtype classification, we generated ranked gene lists for each subtype as discussed in the Methods section (see Table 4 for the statistics). The top 20 genes for each subtype are given in Table 5 (the full results are provided in Additional File 4). The genes are ranked in descending order by their subtype scores (as described in Methods section). To validate the results, we looked at the most recent review on thyroid cancer [34]. Almost all well-observed mutations (see Table 1 in [34]) are identified by our top text mining results, including BRAF, RET, TP53, TRK (NTRK1), RAS (RASA1), PAX8-PPARG, RET/PTC (PTCH1), PTEN and $A K T$. The only missing gene PIK3CA is ranked $30^{\text {th }}$ and $32^{\text {nd }}$ in the lists for FTC and ATC, respectively. This provides solid evidence for the capability of our method in assisting systematic acquisition of knowledge of the molecular biology of thyroid cancer.

We also compared the subtype gene lists generated by text mining to the manually created TCGDB database 
Table 3 Results of TC-relevance for 50 sampled text-mined genes not in Gene2PubMed.

\begin{tabular}{|c|c|c|c|c|c|c|c|}
\hline Gene & Freq. & Related to TC? & PMID & Gene & Freq. & Related to TC? & PMID \\
\hline ENO2 & 78 & YES & 6342746 & MUC5B & 2 & $\mathrm{NO}$ & N/A \\
\hline SYP & 37 & YES & 11740050 & $A D A$ & 2 & YES & 5694958 \\
\hline$A L B$ & 36 & YES & 591616 & MIR34A & 2 & YES & 24220341 \\
\hline CDK2 & 20 & YES & 23895847 & MAPK 10 & 2 & YES & 15619007 \\
\hline$S H B G$ & 12 & YES & 12705335 & $A P O D$ & 2 & YES & 14764826 \\
\hline$\angle M O D 1$ & 9 & YES & 17914110 & WNT7A & 1 & YES & 16676402 \\
\hline P4HB & 7 & NO & N/A & TSHZ3 & 1 & $\mathrm{NO}$ & N/A \\
\hline$S L C 2 A 3$ & 7 & YES & 18571834 & PMS2L11 & 1 & YES & 21606360 \\
\hline THY1 & 7 & NO & N/A & $F H$ & 1 & NO & N/A \\
\hline PCSK2 & 6 & YES & 18661512 & NQO2 & 1 & YES & 23918565 \\
\hline TNFRSFIOB & 6 & YES & 22113498 & MAP3K5 & 1 & YES & 20410161 \\
\hline$A O C 1$ & 5 & YES & 191838 & HYOU1 & 1 & YES & 20719828 \\
\hline ETS1 & 5 & YES & 11280797 & VCP & 1 & YES & 16189643 \\
\hline$A F P$ & 4 & YES & 12428567 & NDRG4 & 1 & $\mathrm{NO}$ & N/A \\
\hline CD14 & 4 & YES & 11389034 & CLDN3 & 1 & YES & 21606360 \\
\hline STAT5A & 3 & YES & 21136677 & COX6A1 & 1 & YES & 23569218 \\
\hline STMN1 & 3 & YES & 15613457 & RAPGEF3 & 1 & YES & 11375794 \\
\hline PRKAA2 & 3 & YES & 24196587 & GNA11 & 1 & YES & 24137342 \\
\hline TAM & 3 & YES & 8077333 & MIR603 & 1 & $\mathrm{NO}$ & N/A \\
\hline CTLA4 & 3 & YES & 18505566 & TAT & 1 & $\mathrm{NO}$ & N/A \\
\hline APOE & 2 & YES & 17690558 & $M L N$ & 1 & YES & 18844033 \\
\hline HBEGF & 2 & YES & 23917679 & FKBP4 & 1 & YES & 22612312 \\
\hline SNAP25 & 2 & YES & 18813355 & EPASI & 1 & YES & 20578836 \\
\hline TRHR & 2 & YES & 23781307 & MFI2 & 1 & YES & 8090582 \\
\hline YY1 & 2 & YES & 23690926 & KLF4 & 1 & YES & 23301671 \\
\hline
\end{tabular}

The PMID column shows an example of document that reports evidence that a given gene is linked to TC, or N/A if we were not able to find support for the relationship.

(see Table 6): only a small number of genes for each subtype were missed by our method. The majority of the genes in the subtype lists from TCGDB have been successfully retrieved by our method, along with a number of candidates for further manual curation.

Figure 5 shows a Venn diagram of gene lists shared between the four subtypes. Compared to Figure 3, we observe a much higher degree of overlap among subtypes. This indicates that a considerable number of common genes are investigated across different subtypes but in separate studies. Meanwhile, each subtype is still characterized by a significant number of unique genes.

\section{Pathway mention recognition results}

Table 7 presents the top 20 pathways for each TC subtype (For the complete text mining results for $\mathrm{TC}$ related pathways, please refer to Additional File 5). The most frequently mentioned pathways are typical biological processes involved in most cancers, including apoptosis, angiogenesis, cell cycle, etc.

To validate these results, we compiled a list of pathways from the most recent review on the molecular biology of thyroid cancers mentioned above [34]. The list includes five pathways: the mitogen-activated protein kinase (MAPK) pathway, the phosphatidylinositol 3-kinase (PI3K)/Akt signaling pathway, the Wnt/betacatenin pathway, the NF-kB pathway and the HypoxiaInducible Factor (HIF)-1 $\alpha$ pathway. Except for the HIF-1 $\alpha$ pathway, all other pathways are present in the top 20 pathways extracted for subtypes by text mining (see Table 8). The HIF-1 $\alpha$ pathway was reported to be a potential therapeutic target for thyroid cancer [35] and

Table 4 Number of genes extracted for each TC subtype.

\begin{tabular}{|c|c|c|c|c|c|}
\hline & PTC & ATC & FTC & MTC & TOTAL \\
\hline \#Genes & 1256 & 538 & 791 & 613 & 2834 \\
\hline \%Unique & $38.7 \%$ & $25.5 \%$ & $15.8 \%$ & $40.6 \%$ & \\
\hline
\end{tabular}

\%Unique represents the percentage of (unique) genes that appear only with a given subtype (compared to the total number of genes appearing with that subtype). So, $38.7 \%$ of genes related to PTC are unique to PTC i.e. appear only with PTC and not with other subtypes. 
Table 5 Top 20 genes for the four thyroid cancer subtypes as extracted from the literature.

\begin{tabular}{|c|c|c|c|c|c|c|c|c|}
\hline \multirow[b]{2}{*}{ Rank } & \multicolumn{2}{|c|}{ PTC } & \multicolumn{2}{|c|}{ ATC } & \multicolumn{2}{|c|}{ FTC } & \multicolumn{2}{|c|}{ MTC } \\
\hline & GENE & $S_{E}^{P}$ & GENE & $S_{E}^{A}$ & GENE & $S_{E}^{F}$ & GENE & $S_{E}^{M}$ \\
\hline$\# 1$ & $\mathrm{TG}$ & 5471 & TP53 & 645 & $\mathrm{TG}$ & 4246 & CALCA & 6173 \\
\hline$\# 2$ & RET & 4916 & $\mathrm{TG}$ & 424 & TSHB & 1039 & RET & 3880 \\
\hline \#3 & BRAF & 2582 & AKT1 & 274 & RET & 585 & SST & 1016 \\
\hline$\# 4$ & PTCH1 & 1829 & CDKN1A & 231 & PAX8 & 453 & $\mathrm{TG}$ & 547 \\
\hline$\# 5$ & TSHB & 1175 & EGFR & 228 & TSHR & 435 & CEACAM3 & 462 \\
\hline$\# 6$ & NCOA4 & 1115 & CASP3 & 213 & BRAF & 419 & GAST & 367 \\
\hline \#7 & RASA1 & 914 & BRAF & 209 & RASA1 & 392 & CEACAM19 & 365 \\
\hline \#8 & TP53 & 819 & RET & 179 & PPARG & 374 & $\mathrm{CHGA}$ & 353 \\
\hline \#9 & LGALS3 & 693 & PAX8 & 151 & LGALS3 & 364 & POMC & 334 \\
\hline$\# 10$ & KRT19 & 662 & RASA1 & 151 & TP53 & 333 & TXK & 325 \\
\hline$\# 11$ & MAPK1 & 658 & $\mathrm{BCL2}$ & 144 & CALCA & 272 & ENO2 & 260 \\
\hline$\# 12$ & TSHR & 570 & CCND1 & 141 & TPO & 266 & NTRK1 & 224 \\
\hline$\# 13$ & CCDC6 & 557 & VIM & 137 & PPBP & 235 & RASA1 & 209 \\
\hline$\# 14$ & NTRK1 & 422 & VEGFA & 121 & AKT1 & 222 & TP53 & 206 \\
\hline$\# 15$ & HBE1 & 386 & NKX2-1 & 120 & KRT19 & 193 & EGFR & 177 \\
\hline$\# 16$ & NKX2-1 & 384 & CALCA & 119 & PTEN & 174 & ELL & 177 \\
\hline$\# 17$ & AKT1 & 371 & PARP1 & 111 & EGF & 173 & AKT1 & 169 \\
\hline$\# 18$ & VEGFA & 369 & TXK & 111 & PPARA & 167 & $\mathrm{KDR}$ & 162 \\
\hline$\# 19$ & PPBP & 343 & PPARG & 108 & NKX2-1 & 156 & SSTR1 & 161 \\
\hline \#20 & MET & 329 & SLC5A5 & 107 & EGFR & 150 & BCL2 & 147 \\
\hline
\end{tabular}

has been experimentally verified in a couple of cell lines [36]. However, this pathway is absent from the textmined results, even though the reporting paper [36] was included in TC_TEXT corpus. Our method did detect the HIF-1 $\alpha$ pathway mention and found its association with thyroid carcinomas. However, the subtype relevancy scores for that abstract (PMID: 19808899) are low. The reason is that the cell lines used in the study were actually subtype-specific but this information was only available in the full-text. This example suggests the importance and potential of the analysis of full text articles as opposed to abstracts, which is an objective for future work.

For other pathways in Table 7 that have not been mentioned in the above review, we have further investigated whether they are associated with the corresponding subtypes. For instance, the Notch signalling appears in the top 20 pathways for PTC, ATC and MTC but was not mentioned in [34]. We however found direct

Table 6 Text-mined genes compared with the TCGDB database.

\begin{tabular}{lllll}
\hline & PTC & ATC & FTC & MTC \\
\hline \#TCGDB & 60 & 15 & 10 & 20 \\
\#TM Missed & 8 & 2 & 3 & 2 \\
\%Coverage & $86.7 \%$ & $86.7 \%$ & $70 \%$ & $90 \%$ \\
\hline
\end{tabular}

The first row represents the total numbers of genes in TCGDB associated to a given subtype. The second row represents the number of genes missed by text mining. The final row is the coverage: (\#TCGDB-\#TM Missed) / \#TCGDB. literature evidence that links this pathway to TC: the Notch pathway has crosstalk with the MAPK pathway and affects the PTC proliferation [37], and the activation of the Notch signalling has been identified as the potential therapeutic strategy for ATC [38]. We followed this procedure for each pathway in Table 7: an example PMID is given in the "Evidence" column, which represents an article that reports the association between the subtype and the pathway (if applicable). Almost all

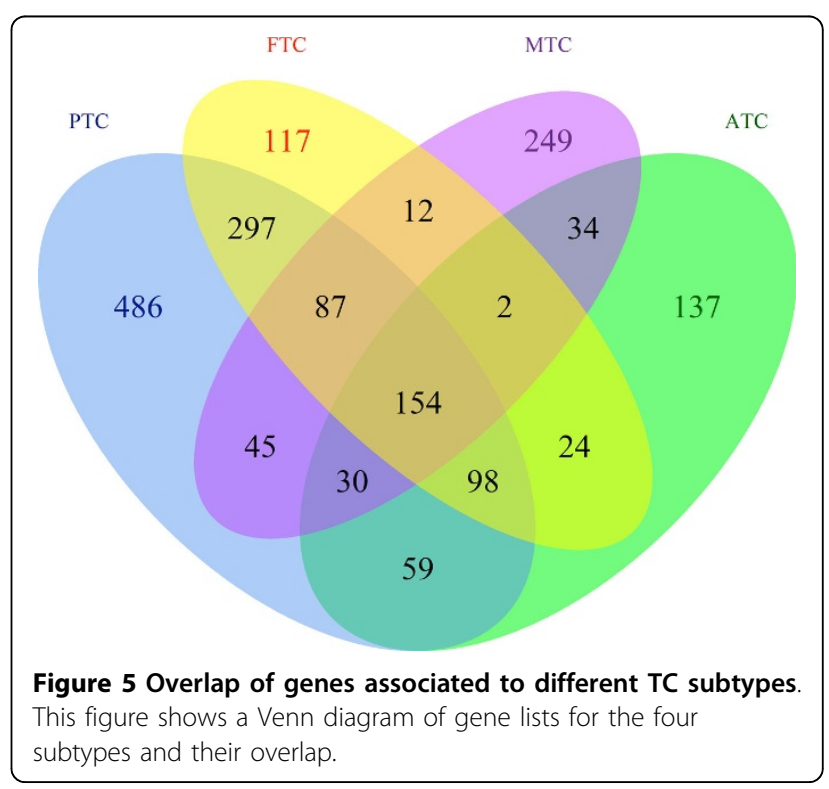


Table 7 Top 20 pathways for the four thyroid cancer subtypes as extracted from the literature.

\begin{tabular}{|c|c|c|c|c|c|c|c|c|}
\hline \multirow[t]{2}{*}{ Top } & \multicolumn{2}{|l|}{ PTC } & \multicolumn{2}{|l|}{ ATC } & \multicolumn{2}{|l|}{ FTC } & \multicolumn{2}{|l|}{ MTC } \\
\hline & Pathway & Evidence & Pathway & Evidence & Pathway & Evidence & Pathway & Evidence \\
\hline$\# 1$ & Apoptosis & 22120515 & Apoptosis & 20067110 & Apoptosis & 24213562 & Apoptosis & 10614665 \\
\hline \#2 & Cell cycle & 23231932 & Cell cycle & 22688732 & Angiogenesis & 14605010 & Cell cycle & 21973234 \\
\hline \#3 & MAPK pathway & 23544999 & Angiogenesis & 17575107 & Cell cycle & 19190121 & Angiogenesis & 20133461 \\
\hline$\# 4$ & Angiogenesis & 23528368 & PI3K/Akt pathway & 22918703 & PI3K/Akt pathway & 18492751 & RET pathway & 15316058 \\
\hline \#5 & DNA Repair & 21860547 & MAPK pathway & 17989125 & DNA Repair & 22331172 & mTOR pathway & 22136849 \\
\hline \#6 & S Phase & 22329804 & S Phase & 18813835 & S Phase & 2874658 & S Phase & 18791128 \\
\hline \#7 & PI3K/Akt pathway & 22744707 & M Phase & 22399519 & MAPK pathway & 18492751 & Focal Adhesion & 12850460 \\
\hline \#8 & Glucose transport & 21606885 & NF-kB pathway & 19158360 & PI3K pathway & 23128507 & MAPK pathway & 15746253 \\
\hline \#9 & PI3K pathway & 20804548 & Glucose transport & 12667615 & Focal Adhesion & 20225271 & $\begin{array}{l}\text { Hedgehog }(\mathrm{Hh}) \\
\text { pathway }\end{array}$ & 23410206 \\
\hline$\# 10$ & $\begin{array}{l}\text { Wnt/beta-catenin } \\
\text { pathway }\end{array}$ & 23261982 & Focal Adhesion & 19293266 & Oxidative Stress & 22331172 & Notch1 pathway & 18520232 \\
\hline$\# 11$ & TGF-beta pathway & 21874046 & Wnt pathway & 15650354 & TGF-beta pathway & 10942134 & bone remodeling & 6611007 \\
\hline$\# 12$ & mTOR pathway & 21822208 & Glycolysis & 3155492 & Glucose transport & 16273245 & mRNA Processing & 2582437 \\
\hline$\# 13$ & Oxidative Stress & 9774495 & Notch1 pathway & 23594881 & $\begin{array}{l}\text { thyroid hormone } \\
\text { production }\end{array}$ & N/A & Raf-1 pathway & 17363508 \\
\hline$\# 14$ & MAPK/ERK pathway & 22426956 & G1 Phase & 9038381 & Glucose metabolism & 19433487 & PI3K pathway & 17188151 \\
\hline$\# 15$ & MEK/ERK pathway & 20629553 & mTOR pathway & 20689131 & cAMP pathway & N/A & Notch pathway & 20182588 \\
\hline$\# 16$ & cAMP pathway & 21479404 & $\begin{array}{l}\text { Hedgehog (Hh) } \\
\text { pathway }\end{array}$ & 23860623 & $\begin{array}{l}\text { thyroid hormone } \\
\text { biosynthesis }\end{array}$ & $\mathrm{N} / \mathrm{A}$ & ERK activation & 21470995 \\
\hline$\# 17$ & Focal Adhesion & 22513979 & STAT3 pathway & 22328572 & Cytokinesis & 15886755 & PI3K/Akt pathway & 23934677 \\
\hline$\# 18$ & Notch pathway & 23544172 & $\begin{array}{l}\text { Wnt/beta-catenin } \\
\text { pathway }\end{array}$ & 17218945 & Hedgehog (Hh) pathway & N/A & Glucose transport & 9426419 \\
\hline$\# 19$ & Glycolysis & 23846818 & p21 pathway & 22918703 & Glycolysis & N/A & EGFR pathway & 22025146 \\
\hline$\# 20$ & Glucose metabolism & 20473281 & $\mathrm{Rb} / \mathrm{E} 2 \mathrm{~F}$ pathway & 15118916 & VEGF pathway & 18509004 & Glycolysis & 3155492 \\
\hline
\end{tabular}

"Evidence" column presents one example PMID that reports a given relationship (N/A: Not Applicable if there is no evidence)

pathways in the top 20 list are supported by literature evidence: only for five pathways in the FTC list we were not able to find support in the literature. This demonstrates the potential of our work for the systematic and comprehensive acquisition of knowledge of thyroid cancer.

\section{Conclusions}

In this paper we presented an approach to finding molecular information associated with different subtypes of thyroid cancer. We developed a method for subtype classification and performed text mining to identify genes and biological pathways associated with each subtype. The generated gene and pathway lists provide a comprehensive compendium of the key molecular information related to thyroid cancer subtypes.

The data and results from our study form the basis for a further comprehensive analysis of the molecular biology of thyroid cancer, enriched by subtypes. Based on the molecular differences between subtypes revealed in our study, biologists can look for better diagnostic biomarkers. In addition, the molecular information can be used to build molecular networks of thyroid cancer subtypes, which would enable further systems biology analyses and stimulate the development of targeted therapeutics.

Table 8 Reviewed TC related pathways and text-mined evidence for each subtype.

\begin{tabular}{lccccc}
\hline Pathway & \multicolumn{2}{c}{ Related to TC? } & \multicolumn{3}{c}{ Evidence PMIDs } \\
\cline { 3 - 6 } & & PTC & ATC & FTC & MTC \\
\hline MAPK pathway & YES & 16896265 & 16410725 & 21196179 & 23934677 \\
PI3K/Akt signaling pathway & YES & 18000091 & 22918703 & 17426084 & 23329180 \\
Wnt/beta-catenin pathway & YES & 22204713 & 17218945 & 18727708 & N/A \\
NF-kB pathway & YES & 23528368 & 19885592 & 16314832 & N/A \\
HIF-1 $\alpha$ pathway & YES & N/A & N/A & N/A & N/A \\
\hline
\end{tabular}




\section{Availability}

The code and data files are available at https:/github.com/ chengkun-wu/GenesThyCan.

\section{Additional material}

Additional File 1: TC_TEXT PMID list. The PMIDs in the TC_TEXT

corpus are listed in this .txt file, one PMID per line.

Additional File 2: SC_GOLD PMID list. The .txt file is the gold standard derived from the MeSH Supplementary Concepts for thyroid cancer. It consists of 1,213 PMIDs and their corresponding thyroid cancer SCs. The values in the file are tab separated.

Additional File 3: Classification results with scores and subtype labels. The .xlsx (Excel) file contains the classification results on the TC_TEXT corpus with subtype relevancy scores and predicated subtype labels. If a document is not related to TC at all, it is given the label "NON"; if a document is related to TC but without a specific focus on one or multiple subtypes, it is given the label "TC"; if a document is assigned multiple subtype labels, the labels are separated by '

Additional File 4: Text-mined gene results. The .xlsx (Excel) file contains the text mining results for TC-related genes. The file contains a mapping between TC-related documents and their associated genes, categorized by subtypes.

Additional File 5: Text-mined pathway results. The .xlsx (Excel) file contains the text mining results for TC-related pathways. The file contains a mapping between TC-related documents and their associated pathways, categorized by subtypes.

\section{List of abbreviations}

NER: Named Entity Recognition; PMID: PubMed ID; TC: Thyroid Cancer; PTC: Papillary Thyroid Cancer; FTC: Follicular Thyroid Cancer; ATC: Anaplastic Thyroid Cancer; MTC: Medullary Thyroid Cancer; DTC: Differentiated Thyroid Cancer; WDTC: Well-Differentiated Thyroid Cancer; MeSH: Medical Subject Headings; SC: Supplementary Concept; TCGDB: Thyroid Cancer and Disorder Gene Database; SK: Subtype Keyword; AK: Anatomy Keyword; MK: Malignancy Keyword; TP: True Positives; FN: False Negatives; FP: False Positives;

\section{Competing interests}

The authors declare that they have no competing interests.

\section{Authors' contributions}

CW developed the system for the detection on the subtype-genes/pathways association, and drafted the manuscript. GN provided support and guidance from the text mining perspective and JMS from the systems biology perspective. GB provided expertise on thyroid cancer. GN and JMS conceived and supervised the project. CW, JMS, GB and GN wrote the manuscript. All authors read and approved the final manuscript.

\section{Acknowledgements}

CW is co-funded by UK Biotechnology and Biological Sciences Research Council (BBSRC), the University of Manchester and the Chinese Scholarship Council (CSC) for his PhD study in the Doctoral Training Centre for Integrative Systems Biology at the University of Manchester. GN acknowledges support from the Serbian Ministry of Education and Science (projects III44006; III47003) and the Health eResearch Centre (HeRC).

\section{Declarations}

Publication of this article was funded by the UK Biotechnology and Biological Sciences Research Council (BBSRC) through the Doctoral Training Centre for Integrative Systems Biology at the University of Manchester. This article has been published as part of BMC Medical Genomics Volume 7 Supplement 3, 2014: Thirteenth International Conference on Bioinformatics (InCoB2014): Medical Genomics. The full contents of the supplement are available online at http://www.biomedcentral.com/bmcmedgenomics/ supplements/7/S3.

\section{Authors' details}

${ }^{1}$ Faculty of Life Sciences, University of Manchester, Manchester, M13 9PT, UK. ${ }^{2}$ Doctoral Training Centre in Integrative Systems Biology, University of Manchester, 131 Princess Street, Manchester M1 7DN, UK. ${ }^{3}$ Manchester Institute of Biotechnology, 131 Princess Street, Manchester M1 7DN, UK. ${ }^{4}$ Department of Endocrinology, Christie Hospital, University of Manchester, Wilmslow Road, Manchester M20 4BX, UK. ${ }^{5}$ Experimental and Clinical Endocrinology, Med Clinic I, University of Luebeck Ratzeburger Allee 160 D23538, Lübeck, Germany. ${ }^{6}$ School of Computer Science, University of Manchester, Manchester M13 9PL, UK. ${ }^{7}$ Health e-Research Centre (HeRC), Manchester M13 9PL, UK.

Published: 8 December 2014

\section{References}

1. Nix P, Nicolaides A, Coatesworth AP: Thyroid cancer review 1: presentation and investigation of thyroid cancer. International Journal of Clinical Practice 2005, 59:1340-1344.

2. Ito Y, Nikiforov YE, Schlumberger M, Vigneri R: Increasing incidence of thyroid cancer: controversies explored. Nature Reviews Endocrinology 2013, 9:178-184.

3. Rodríguez-Rodero S, Fernández AF, Fernández-Morera JL, Castro-Santos P, Bayon GF, Ferrero C, Urdinguio RG, Gonzalez-Marquez R, Suarez C, Fernández-Vega I, Fresno Forcelledo MF, Martínez-Camblor P, Mancikova V, Castelblanco E, Perez M, Marrón PI, Mendiola M, Hardisson D, Santisteban P, Riesco-Eizaguirre G, Matías-Guiu X, Carnero A, Robledo M, DelgadoÁlvarez E, Menéndez-Torre E, Fraga MF: DNA methylation signatures identify biologically distinct thyroid cancer subtypes. J Clin Endocrinol Metab 2013, 98:2811-2821.

4. Lalami Y, Awada A: Recurrent thyroid cancer: a molecular-based therapeutic breakthrough. Curr Opin Oncol 2011, 23:235-240.

5. Xing $M$, Haugen BR, Schlumberger M: Progress in molecular-based management of differentiated thyroid cancer. Lancet 2013, 381:1058-1069.

6. Sipos JA, Shah MH: Thyroid cancer: emerging role for targeted therapies. Ther Adv Med Oncol 2010, 2:3-16.

7. Antonelli A, Fallahi P, Ferrari SM, Ruffilli I, Santini F, Minuto M, Galleri D, Miccoli P: New targeted therapies for thyroid cancer. Curr Genomics 2011, 12:626-631.

8. Nikiforova MN, Nikiforov YE: Molecular genetics of thyroid cancer: implications for diagnosis, treatment and prognosis. Expert Rev Mol Diagn 2008, 8:83-95.

9. Russo D, Damante G, Puxeddu E, Durante C, Filetti S: Epigenetics of thyroid cancer and novel therapeutic targets. J Mol Endocrinol 2011 , 46:R73-R81.

10. Mulligan LM: RET revisited: expanding the oncogenic portfolio. Nat Rev Cancer 2014, 14:173-186.

11. Hunter L, Cohen KB: Biomedical language processing: what's beyond PubMed? Mol Cell 2006, 21:589-594.

12. Ananiadou S, Kell DB, Tsujii J-I: Text mining and its potential applications in systems biology. Trends in Biotechnology 2006, 24:571-579.

13. Ananiadou S, Pyysalo S, Tsujii J, Kell DB: Event extraction for systems biology by text mining the literature. Trends in Biotechnology 2010 28:381-390.

14. Neves ML, Carazo J-M, Pascual-Montano A: Moara: a Java library for extracting and normalizing gene and protein mentions. $B M C$ Bioinformatics 2010, 11:157.

15. Hakenberg J, Gerner M, Haeussler M, Solt I, Plake C, Schroeder M, Gonzalez G, Nenadic G, Bergman CM: The GNAT library for local and remote gene mention normalization. Bioinformatics 2011, 27:2769-2771.

16. Wu C, Schwartz J-M, Nenadic G: PathNER: a tool for systematic identification of biological pathway mentions in the literature. $B M C$ Syst Biol 2013, 7:S2

17. Bjorne J, Ginter F, Pyysalo S, Tsujii J, Salakoski T: Complex event extraction at PubMed scale. Bioinformatics 2010, 26:i382-i390.

18. Gerner M, Sarafraz F, Bergman CM, Nenadic G: BioContext: an integrated text mining system for large-scale extraction and contextualisation of biomolecular events. Bioinformatics 2012, 28:2154-2161. 
19. Rappaport N, Nativ N, Stelzer G, Twik M, Guan-Golan Y, Stein TI, Bahir I, Belinky F, Morrey CP, Safran M, Lancet D: MalaCards: an integrated compendium for diseases and their annotation. Database (Oxford) 2013, 2013:bat018.

20. Maglott D, Ostell J, Pruitt KD, Tatusova T: Entrez Gene: gene-centered information at NCBI. Nucleic Acids Res 2011, , 39 Database: D52-7.

21. Kanehisa M, Goto S, Sato Y, Furumichi M, Tanabe M, Kanehisa M, Goto S, Sato $Y$, Furumichi M, Tanabe M: KEGG for integration and interpretation of large-scale molecular data sets. Nucleic Acids Res 2011, 40:D109-D114.

22. Mizuno S, lijima R, Ogishima S, Kikuchi M, Matsuoka Y, Ghosh S, Miyamoto T, Miyashita A, Kuwano R, Tanaka H: AlzPathway: a comprehensive map of signaling pathways of Alzheimer's disease. BMC Syst Biol 2012, 6:52.

23. Chun H-W, Tsuruoka Y, Kim J-D, Shiba R, Nagata N, Hishiki T, Tsujii J: Automatic recognition of topic-classified relations between prostate cancer and genes using MEDLINE abstracts. BMC Bioinformatics 2006, 7:S4

24. Tsuruoka Y, Tsujii J, Ananiadou S: FACTA: a text search engine for finding associated biomedical concepts. Bioinformatics 2008, 24:2559-2560.

25. Frijters R, Heupers B, van Beek P, Bouwhuis M, van Schaik R, de Vlieg J, Polman J, Alkema W: CoPub: a literature-based keyword enrichment tool for microarray data analysis. Nucleic Acids Res 2008, 36 Web Server: 1-5.

26. Kim J, So S, Lee H, Park JC, Kim J-J, Lee H: DigSee: Disease gene search engine with evidence sentences (version cancer). Nucleic Acids Res 2013, 41 Web Server: W510-7.

27. Sebastiani F: Machine learning in automated text categorization. ACM Computing Surveys (CSUR) 2002, 34:1-47.

28. Gerner M, Nenadic G, Bergman CM: LINNAEUS: a species name identification system for biomedical literature. BMC Bioinformatics 2010, 11:85.

29. Neves M, Chagoyen M, Carazo JM, Pascual-Montano A: CBR-Tagger: a casebased reasoning approach to the gene/protein mention problem. Association for Computational Linguistics; 2008.

30. Leaman R, Gonzalez G: BANNER: an executable survey of advances in biomedical named entity recognition. Pac Symp Biocomput 2008, 652-663.

31. Kamburov A, Stelzl U, Lehrach H, Herwig R: The ConsensusPathDB interaction database: 2013 update. Nucleic Acids Res 2013, , 41 Database: D793-800

32. Busaidy NL, Cabanillas ME: Differentiated thyroid cancer: management of patients with radioiodine nonresponsive disease. Journal of Thyroid Research 2012, 2012:618985.

33. Grande E, Díez JJ, Zafon C, Capdevila J: Thyroid cancer: molecular aspects and new therapeutic strategies. Journal of Thyroid Research 2012, 2012:847108.

34. Omur O, Baran Y: An update on molecular biology of thyroid cancers. Crit Rev Oncol Hematol 2014, 90:233-252.

35. Burrows N, Babur M, Resch J, Williams KJ, Brabant G: Hypoxia-inducible factor in thyroid carcinoma. Journal of Thyroid Research 2011, 2011:762905.

36. Burrows N, Resch J, Cowen RL, Wasielewski von R, Hoang-Vu C, West CM, Williams KJ, Brabant G: Expression of hypoxia-inducible factor 1 in thyroid carcinomas. Endocrine Related Cancer 2010, 17:61-72

37. Yamashita AS, Geraldo MV, Fuziwara CS, Kulcsar MAV, Friguglietti CUM, da Costa RB, Baia GS, Kimura ET: Notch pathway is activated by MAPK signaling and influences papillary thyroid cancer proliferation. Trans/ Oncol 2013, 6:197-205.

38. Yu X-M, Jaskula-Sztul R, Ahmed K, Harrison AD, Kunnimalaiyaan M, Chen H: Resveratrol induces differentiation markers expression in anaplastic thyroid carcinoma via activation of Notch1 signaling and suppresses cell growth. Mol Cancer Ther 2013, 12:1276-1287.

39. Sippel RS, Kunnimalaiyaan M, Chen $\mathrm{H}$ : Current management of medullary thyroid cancer. Oncologist 2008, 13:539-547.

doi:10.1186/1755-8794-7-S3-S3

Cite this article as: Wu et al:: Molecular profiling of thyroid cancer subtypes using large-scale text mining. BMC Medical Genomics 2014 7(Suppl 3):S3.

\section{Submit your next manuscript to BioMed Central and take full advantage of:}

- Convenient online submission

- Thorough peer review

- No space constraints or color figure charges

- Immediate publication on acceptance

- Inclusion in PubMed, CAS, Scopus and Google Scholar

- Research which is freely available for redistribution

Submit your manuscript at www.biomedcentral.com/submit
Biomed Central 